\title{
MTX gegen Psoriasis: Therapieversuch von zwölf Wochen ist ausreichend
}

\author{
Wie viel Zeit sollte man einer ungenügend ansprechenden Methotrexat- \\ therapie bei Psoriasis geben, bevor man sie als gescheitert beurteilt und den \\ Patienten auf ein anderes Medikament umstellt? Eine Post-hoc-Analyse der \\ CHAMPION-Studie liefert wichtige Hinweise.
}

n der CHAMPION-Studie, einer Vergleichsuntersuchung mit Adalimumab, Methotrexat (MTX) und Placebo, wurde MTX nach einem vorgegebenen Schema auftitriert: In den ersten acht Wochen steigerten Ärzte die Dosis bei allen Patienten von 7,5 auf $15 \mathrm{mg}$ pro Woche. Verbesserte sich der Psoriasis Area and Severity Index (PASI) in dieser Zeit nicht um mindestens $50 \%$, erfolgte in den nächsten vier Wochen die Gabe von 20 mg. Bei PASI-50-Nonrespondern nach zwölf Wochen wurde die Dosis nochmals auf $25 \mathrm{mg}$ erhöht.

Von 103 MTX-Patienten lagen Daten bis zur 16. Woche vor. 40 Patienten wurden als Early Responder (ER) eingestuft ( $\geq$ PASI 50 in Woche 8), 22 als Late Re- sponder (LR, < PASI 50 in Woche 8 und $\geq$ PASI 50 in Woche 12) und 41 als Late Nonresponder (LN, < PASI 50 in Woche 8 und 12).

In der 16. Woche hatte sich der PASI am stärksten in der ER-Gruppe und vergleichbar gut auch in der LR-Gruppe gebessert (im Mittel um 79,9\% bzw. um 69,7\%). Bei der LN-Gruppe betrug die Verbesserung gegenüber dem Ausgangswert nur 24,8\%. Eine PASI-Verbesserung um 75\% erreichten 70\% der Early Responder, $41 \%$ der Late Responder sowie $5 \%$ der Late Nonresponder. Die Mehrheit der Patienten mit einer PASI-75-Response in Woche $16 \mathrm{kam}$ aus der ERGruppe (72\%), 23\% waren aus der LRGruppe.
Nach diesen Ergebnissen hatten nahe$\mathrm{zu}$ alle PASI-75-Responder (bei der Beurteilung in der 16 . Woche) bereits in der achten oder zwölften Woche mit maximal 15-20 mg MTX eine mindestens 50\%ige Verbesserung erfahren. Zudem erfuhren Patienten mit einer PASI-Response von weniger als $50 \%$ in der zwölften Woche auch bei einer weitere Steigerung der MTX-Dosis kaum einen zusätzlichen Nutzen.

Fazit: Werden Psoriasispatienten nach dem CHAMPION-Schema mit MTX behandelt und sprechen nicht gut auf diese Therapie an (PASI-Verbesserung um weniger als 50\%) - so ist es nach den Autoren der vorliegenden Untersuchung sinnvoll, bereits nach zwölf Wochen auf ein anderes Medikament zu wechseln.

Dr. Beate Schuhmacher

Saurat J-H et al. Relationship between methotrexate dosing and clinical response in patients with moderate to severe psoriasis: subanalysis of the CHAMPION study.

BJD 2011; 165: 399-406

\section{Allergien gegen Isopropanol kommen häufiger vor als gedacht}

Isopropanol gilt als eine schwach und nur selten sensibilisierende Substanz. Eine Autorengruppe aus Spanien und Belgien hat nun eine Fallserie präsentiert: So selten, wie bislang oft angenommen, sind allergische Reaktionen auf Isopropylalkohol demnach nicht.

\footnotetext{
$A \mathrm{n}$ der vorliegenden Studie waren 1.450 Patienten beteiligt, die Isopropanol ausgesetzt gewesen waren und sich in den vergangenen 20 Jahren einem Patchtest unterzogen. 44 von ihnen (3\%) zeigten eine allergische Reaktion. Vier Patienten präsentierten sich mit chronischem Handekzem (ein Mechaniker, drei Krankenschwestern). Bei den restlichen 40 Patienten war keine berufliche Exposition erkennbar. 14 von ihnen litten an Ulzera oder Ekzemen der Beine, die übrigen 26 Studienteilnehmer waren an dermatologische Zentren wegen Ekzemen überwiesen worden, die nach dem Gebrauch von isopropanolhaltiger Reinigungs- oder Desinfektionsmitteln beobachtet wurden.
}

Isopropanol ist nach diesen Daten aus allergologischer Sicht keineswegs so harmlos wie bisher angenommen. Dies ist schon deshalb relevant, weil der sekundäre Alkohol häufig für den täglichen Gebrauch empfohlen wird - etwa zum Reinigen der Hände: Die WHO rät für die Handhygiene in medizinischen Einrichtungen zu Substanzen auf alkoholischer Basis. Gerade medizinisches Personal ist in hohem Maß Isopropanol ausgesetzt.

Hergestellt wird Isopropanol (auch als Isopropylalkohol oder 2-Propanol bezeichnet) durch die Hydratisierung von Propen oder Hydrierung von Aceton. Als industrielles Lösungs- und Reinigungsmittel ist es im Gebrauch weit verbreitet und etwa in Lacken, Tinten und Verdünn- nungsmitteln, aber auch in vielen Haushaltsprodukten enthalten.

Es dient außerdem als minder toxische Alternative zu Formaldehyd zum Konservieren sowie als Antiseptikum. In der EU ist Isopropanol auch für die Produktion von Kosmetika zugelassen. Es wird hier als Lösungsmedium, Schauminhibitor, Duftstoff und zur Viskositätskontrolle eingesetzt.

Fazit: Diese Studie, eine große Fallserie zum kontaktallergischen Potenzial von Isopropanol, revidiert das Bild, wonach der sekundäre Alkohol lediglich ein mildes Augen- und Schleimhautirritans darstellt. Die Sensibilisierungsrate betrug bei den Studienteilnehmern immerhin $3 \%$. Zu den gefährdeten Personen gehören vor allem die Angehörigen medizinischer Berufe, die mit Isopropanol praktisch täglich in Berührung kommen.

Dr. Robert Bublak

García-Gavín J et al. Allergic contact dermatitis caused by isopropyl alcohol: a missed allergen? Contact Dermatitis 2011; 65: 101-6 